\title{
Electrostatic-Potential Analysis of Charged Particles by Split-Illumination Electron Holography
}

Toshiaki Tanigaki ${ }^{1,2}$, Zentaro Akase ${ }^{2,3}$, Shinji Aizawa ${ }^{2}$, Hyun Soon Park ${ }^{4}$, Yasukazu Murakami ${ }^{2,3}$, Daisuke Shindo ${ }^{2,3}$ and Hiromitsu Kawase ${ }^{5}$

1. Central Research Laboratory, Hitachi, Ltd., Hatoyama 350-0395, Japan

2. Center for Emergent Matter Science (CEMS), RIKEN, Wako 351-0198, Japan

3. Institute of Multidisciplinary Research for Advanced Materials, Tohoku University, Sendai 980-8577, Japan

4. Department of Materials Science \& Engineering, Dong-A University, Busan 604-714, Republic of Korea

5. System Research \& Development Center, Ricoh Institute of Technology, RICOH Co., Ltd., Kanagawa 224-0035, Japan

Electron holography is a powerful technique for high-resolution evaluation of electromagnetic field distributions in and around materials. Precise electrostatic potential evaluations, however, have been difficult to perform because of the following reasons: The range of Coulomb electrostatic field is much longer than the lateral coherence length of the illuminating electron waves used in electron holography [1]; The incident electrons for observation as well as secondary electrons emitted from the sample can cause changes in electrostatic distributions in and around the sample with low-conductivity [2].

Split-illumination electron holography (SIEH) [3] is a suitable technique for these analyses because it can overcome the problem of lateral coherence length by setting the reference wave away from the sample. In this paper, we report the first practical application of SIEH in the analysis of the electrostatic potential distributions in and around charged insulating materials, particularly model toner samples containing polystyrene particles on a carrier particle used in electrophotography.

Figure 1 shows a typical scanning electron microscopic image of toner particles on a carrier. Note that the image was obtained after holographic observations were performed. The electrostatic potential distributions around the toner particle indicated by an arrow were evaluated using SIEH. Figure 2 shows the schematic of SIEH electron optics. To prevent charging of the insulating samples by electron irradiation during the electron holographic observation, a mask $\mathrm{Cu}$ plate was placed at illuminating system. In addition, adjusting a mask focusing lens placed between the mask and the sample, the focused shadow of the mask was formed exactly on the sample.

The electrostatic potentials were analyzed by phase shift simulation using a model in which the electrostatic potentials had axial symmetry along the axis penetrating the center of the toner and the carrier particles. Figure 3 shows the results: the potential distributions around the toner particles on the carrier were nonuniform [4].

The results obtained by the developed method have deepened our understanding of the potential distributions, which should lead to further improvements in electrophotography. This method is a powerful technique for clarifying the charge distributions of and around various types of insulating materials used in applied physics and engineering. 


\section{References:}

[1] G. Matteucci et al., J. Appl. Phys. 69 (1991) p.1835.

[2] M. Shirai et al., Ultramicroscopy 146 (2014) p.125.

[3] T. Tanigaki et al., Appl. Phys. Lett. 101 (2012) 043101.

[4] T. Tanigaki et al., Appl. Phys. Lett. 104 (2014) 131601.

[5] This research was partly supported by a grant from the Japan Society for the Promotion of Science (JSPS) through the "Funding Program for World-Leading Innovative R\&D on Science and Technology (FIRST Program)” initiated by the Council for Science and Technology Policy (CSTP). This work was partly supported by the Cooperative Research Program of "Network Joint Research Center for Materials and Devices” from the Ministry of Education, Culture, Sports, Science and Technology (MEXT).
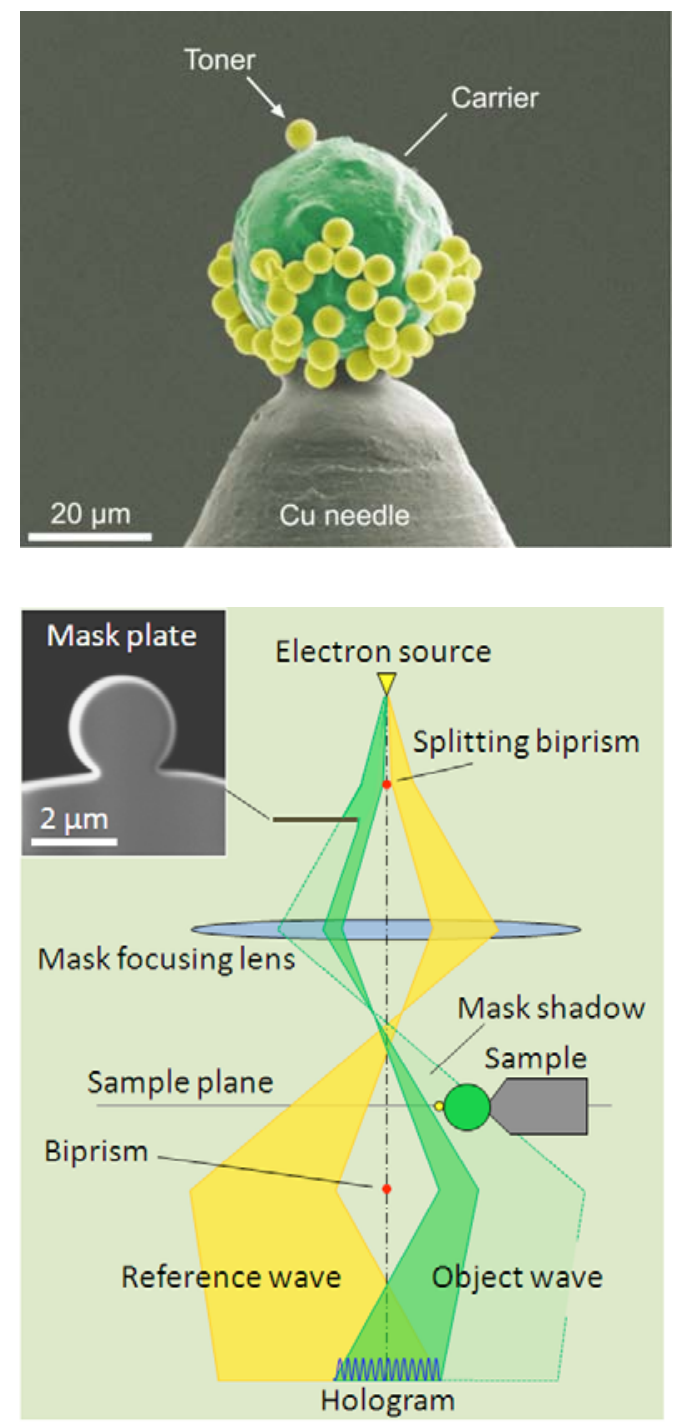

Figure 2. Schematic of SIEH electron optics for observing electrostatic potential distributions of charged insulating materials.
Figure 1. Scanning electron microscopic image of positive toner particles (yellow colored) on a carrier particle (green colored). Electrostatic potential distributions around the toner particle including area near carrier particle were analyzed by electron holography and electron phase shift simulation.

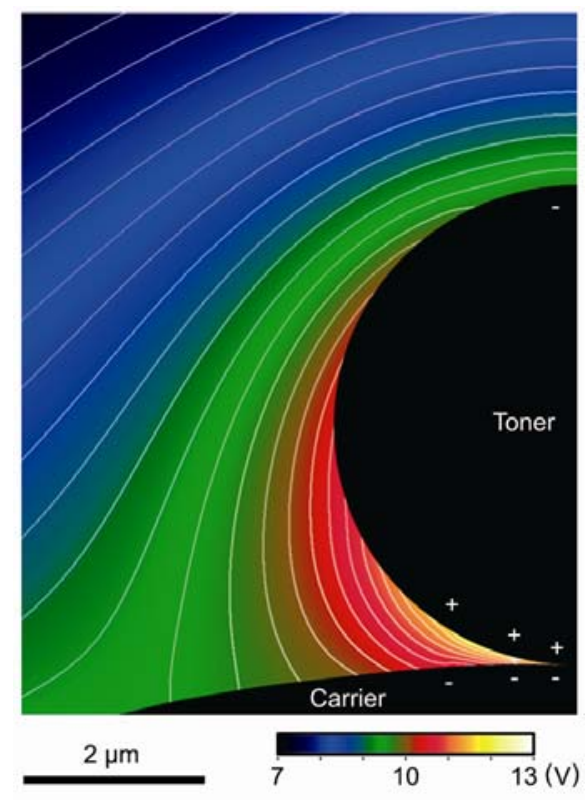

Figure 3. Analyzed electrostatic potential distributions around positive toner particle in the plane perpendicular to the electron beam (cross-sectional view). 